\title{
Metrópolis y Globalización: un Panorama de las Zonas Metropolitanas del Occidente de México
}

\author{
Marco A. Medina Ortega $a^{(*)}$ \\ Rosario Cota Yáñez(*)
}

\begin{abstract}
Resumen: Este artículo hace referencia al proceso de urbanización que ha sufrido el Occidente de México, una característica que ha experimentado la misma América Latina como consecuencia de los cambios de las relaciones económicas mundiales surgidas de un interminable proceso de globalización entre diversas actividades de producción y consumo de los distintos países. Esto ha dado como consecuencia una reconfiguración en las tendencias de urbanización. En la actualidad los procesos de urbanización se han desvinculado del crecimiento de la población como el principal factor que condicionaba su crecimiento. Han contribuido de manera importante a estos cambios los avances tecnológicos y la orientación de la producción enfocada al comercio internacional, los cuales han modificado la orientación geográfica industrial y de los servicios dentro de un determinado territorio.
\end{abstract}

Palabras claves: Zonas Metropolitanas, Occidente de México, Urbanización, Dinámica de Crecimiento.

\begin{abstract}
This paper concerns the process of urbanization that the West of Mexico has undergone, as indeed has Latin America in general, following the changes in economic relations worldwide induced by the endless globalization of various production and consumption activities in different countries. The process called globalization has led to a reconfiguration of the tendencies involved in urbanization, which is no longer linked to population growth as the principal factor determining the growth of cities. Technological progress and the orientation of production to international trade have been important factors in bringing about these changes, as they have modified the geographical disposition of industry and of services within specific territories.
\end{abstract}

Keywords: Metropolitan Areas, Research Scheme, West of Mexico, Urbanization, Dynamics of Growth.

(*) Profesor-investigador de la Universidad de Guadalajara. Departamento de Estudios Regionales. E-mail: <mrmedina@cucea.udg.mx>.

(**) Profesor-investigador de la Universidad de Guadalajara, Centro Universitario de Ciencias Económico-Administrativas —CUCEA. Departamento de Estudios Regionales. E-mail:<macotaya@gmail.com>. Recebido em: 18.12.2009 e aceito em: 15.11.2010. 


\section{INTRODUCCIÓN}

Después de un siglo de urbanización y expansión demográfica, América Latina se estrena en el siglo XXI como una de las regiones más urbanizadas del mundo en desarrollo y con ello atestiguamos la configuración de nuevas tendencias urbanas. En América Latina la urbanización tradicionalmente se ha asociado con altos índices de crecimiento demográfico, sin embargo, desde hace algunas décadas la población urbana ha venido aumentado mucho más rápidamente que la población total.

Consideramos importante destacar que las tendencias demográficas se han visto acompañadas por otros fenómenos que han potenciado aún más las tremendas transformaciones que estamos viviendo en cuanto al proceso de urbanización hoy en día. En ese sentido el creciente proceso de globalización, visto como un proceso de reestructuración de la economía internacional, ha venido a introducir importantes cambios en las estructuras espaciales de la producción y el consumo. De manera que la descentralización de la industria hacia espacios periféricos, los avances tecnológicos y la creciente orientación del aparato productivo hacia el comercio mundial contribuyen de manera sustantiva en la cambiante geografía de la industria y de los servicios que actualmente se manifiesta como un proceso cuya expresión territorial puede variar en periodos más cortos de tiempo que en el siglo pasado.

En el conjunto de transformaciones que enfrentan los países de América Latina en cuanto a sus patrones de urbanización, que no son exclusivas de esta región, se destacan aspectos como la considerable disminución de los índices de crecimiento demográfico y urbano; a lo anterior se le suma que las ciudades de mayor tamaño muestran una tendencia hacia la baja en su explosivo crecimiento que se expresa a través de menores tasas de crecimiento que en algunos casos se ha considerado como un fenómeno de inversión en su crecimiento ${ }^{(1)}$; y, por último, el surgimiento de un conjunto de ciudades de menor tamaño que manifiestan no solo un intenso crecimiento poblacional sino también una creciente importancia de estas que nos llevan a considerar el posible surgimiento y consolidación de una red urbana con una jerarquía más equilibrada entre las ciudades.

De manera que en el presente trabajo nos proponemos reflexionar en torno al proceso de metropolización en el contexto de la globalización para, posteriormente, revisar el conjunto de zonas metropolitanas del occidente de México en cuanto a su dinámica de crecimiento en los últimos años y por último reflexionar en torno a la propuesta de una agenda de investigación para las zonas metropolitanas de esta región del país.

(1) Cambios que se constituye en una paradoja en el proceso de urbanización ya que por primer ocasión se presenta una expansión de la ciudad generada al margen de los comportamientos demográficos, esto es, mientras que la población de las grandes ciudades se mantenía estable o decrecía, la expansión del área urbana se incrementaba mediante una dispersión de la urbanización sin umbrales territoriales fácilmente observables que De Mattos señala como metropolización expandida (DE MATTOS, 2002). 


\section{EL PROCESO DE METROPOLIZACIÓN}

La evolución del espacio urbano está fuertemente ligado a procesos de carácter económico. En este proceso evolutivo sin lugar a dudas la revolución industrial se constituye como un parteaguas en el desarrollo de la ciudad, al grado de caracterizarla como el espacio industrial por excelencia que permitió el crecimiento de una actividad productiva tan intensa y con ello una expansión urbana sin precedentes. Este hecho lo considera Miret como el primer antecedente del fenómeno de metropolización cuyo origen se remontan al Londres del siglo XIX y que posteriormente se extiende por numerosas capitales de Europa y América a principios del siglo XX. A partir de este situación las capitales políticas o económicas van a conocer un crecimiento urbano sin precedentes, en el cual se combinan las necesidades de emigrar de las poblaciones rurales y la fuerte demanda de población por parte de la naciente industria (MIRET, 2001).

Se considera que la segunda fase del proceso de metropolización empieza con la segunda guerra mundial, prolongándose hasta los años setenta, durante este periodo las ciudades incorporan una serie de funciones que conjuntamente con su creciente incremento demográfico les otorgan la capacidad de generar por sí mismas su crecimiento. Hay que destacar que durante este periodo la constitución de áreas metropolitanas surge como una necesidad en el ámbito de la planeación de la ciudad a través del establecimiento de organismos de gestión urbana para la atención de fenómenos urbanos como la vialidad, el transporte, la vivienda, el agua, el drenaje, la seguridad, entre otros aspectos (MIRET, 2001).

Durante este periodo ${ }^{(2)}$ las grandes industrias localizadas en grandes ciudades necesitaron de una abundante mano de obra que se alojó en las periferias urbanas, en ese sentido el tejido urbano periférico adquirió rápidamente un carácter de continuidad constituyendo las denominadas coronas metropolitanas o anillos exteriores. En ese sentido empezamos a atestiguar fenómenos como la sobreposición de un conjunto de ciudades que inicialmente crecieron separadas (conurbación), o casos en que un centro va originando nuevos y diversos espacios periféricos dependientes (satélites), fenómenos que hoy en día se constituyen en antecedentes del proceso de metropolización.

La tercer fase del proceso de metropolización surge a partir de los años setenta muy de la mano de la actual fase del proceso de globalización, más específicamente del proceso de reestructuración económica, tan es así que algunos autores hacen referencia a la existencia de grandes metrópolis como ciudades posfordistas; en ese sentido el estudio de las metrópolis se hace más complejo entre otros aspectos por que su contenido se amplia al grado que en los estudios sobre el crecimiento urbano las metrópolis adquieren una singular preponderancia (MIRET, 2001).

De manera que la globalización ha venido ha consolidar la importancia de las ciudades a través de diferentes procesos uno de ellos es la economía en red, esto es, con la reestructuración económica se incremento la complejidad de las transacciones económicas y con ello la necesidad de fortalecer ciertas funciones en las empresas

(2) Que se corresponde con el auge y consolidación del sistema de producción fordista. 
transnacionales además de una expansión de la rama de los servicios al productor, actividad que no solamente se ha expandido en las empresas transnacionales, sino que se han venido incorporando paulatinamente también en otros sectores y empresas.

Al respecto De Mattos (2001) considera que el espacio donde se han concentrado los servicios especializados y las actividades económicas son las metrópolis, lo que para el significa como un proceso de urbanización de la economía, y en ese sentido las ciudades se han consolidado como lo nodos en torno a los que se articula la actual dinámica de crecimiento. De manera que las ciudades en esta etapa pueden incorporar actividades tanto de control o dirección, además de ser sitios de producción manufacturera de carácter posfordista (en alguna de las fases del proceso productivo que pueden ser intensivas en capital o en mano de obra o una combinación de ambas), y también pueden incorporar actividades del sector financiero y servicios especializados. Conforme este proceso se ha extendido por el planeta, diversas ciudades de los países involucrados se han constituido como los espacios elegidos para el establecimiento de diversas fases del proceso productivo, configurado, en algunos casos, y reconfigurado, en otros, la base económica de las ciudades.

Sin embargo y a pesar de que este es un proceso general que presentan las ciudades tanto de países desarrollados como en desarrollo, no hay que dejar de lado la existencia de ciertas diferencias que presentan las ciudades y que están asociadas con el nivel de desarrollo de los países que las contienen. En ese contexto Adriana Olivares y Daniel González (2004) destacan que no obstante la existencia de características comunes entre las metrópolis a nivel general, estas presentan ciertas especificidades asociadas con el nivel de desarrollo de cada país así como también al de su región. Al respecto señalan que en los países desarrollados los territorios urbanos concentran actividades vinculadas a los servicios avanzados (gestión, dirección y control de la producción a nivel mundial), así como actividades ligadas al sector financiero; mientras que los territorios de países en desarrollo están concentrado básicamente actividades productivas con un nivel de especialización bajo y que suelen hacer un uso intenso de la abundante mano de obra barata que predomina en estas economías (OLIVARES; GONZÁLEZ, $2004)$.

Paradójicamente y a pesar de que en los países en desarrollo se localizan actividades poco especializada, De Mattos considera que en la medida en que las nuevas actividades están generando una creciente demanda de infraestructura urbana se ha intensificado la localización metropolitana de este tipo de actividades contribuyendo al desencadenamiento de "una dinámica de metropolización expandida, en la que progresivamente van ocupando los pueblos y áreas rurales que encuentran a su paso, desbordando una y otra vez sus límites anteriores. De estos incontrolables procesos de suburbanización, en cuya dinámica el automóvil tiene una incidencia decisiva, emergen ciudades de cobertura y alcance regional, de estructura policéntrica y fronteras difusas. (DE MATTOS, 2001, p. 2).

\section{ELEMENTOS DE DEBATE EN LA RELACIÓN METRÓPOLIS/GLOBALIZACIÓN}

Sin lugar a dudas los cambios en el modelo económico están generando efectos diferenciados en los ámbitos social, económico, político y cultural pero también en el 
territorial; dando como resultado que algunas ciudades y territorios se inserten de manera más dinámica al proceso de globalización, en ese orden de ideas nos encontramos, entre los estudios que analizan los efectos urbanos y territoriales de la globalización, muy a menudo la idea del resurgimiento de las grandes ciudades y de su crecimiento (MARKUSEN; SCHROCK, 2006), en gran medida bajo la perspectiva de la expansión metropolitana que como bien señala De Mattos da lugar a una nueva geografía urbana (MATTOS, 2002).

Si bien se acepta lo anterior no hay que dejar de lado la existencia de algunas dudas, entre estas encontramos lo señalado por Markusen y Schrock (2006) quienes en un análisis sobre crecimiento urbano de 50 grandes áreas metropolitanas de los Estados Unidos en el periodo 1980-2000, argumentan que a pesar de la acelerada integración del mercado mundial no existe un resurgimiento generalizado de las grandes ciudades; además entre sus hallazgos señalan la existencia de economías urbanas que aunque no son de gran tamaño están prosperando con base en la especialización. Para el caso de América latina De Mattos, en un trabajo sobre las transformaciones de las ciudades latinoamericanas derivadas de los ¿impactos de la globalización?, señala que si bien es aceptado de manera común que los principales cambios en las metrópolis son consecuencia de las transformaciones asociadas a la globalización, existen discusiones entre especialistas en cuestiones urbanas que ponen en duda esta relación causal ${ }^{(3)}$ (DE MATTOS, 2002).

Por otra parte entre los elementos más destacados o con mayor atención en cuanto a las transformaciones metropolitanas derivadas del proceso de globalización encontramos la existencia de ciudades globales y el proceso de terciarización de la base económica de estas. En The Global City. New York, London, Tokio (2001) Saskia Sassen propone la existencia de una red global de ciudades que ejercen funciones estratégicas para las operaciones económicas globales, entre las que destaca las tareas de dirección, gestión y control que han provocado la concentración de lo que se denomina como las actividades más modernas del terciario avanzado entre las que destacan los servicios al productor, servicios financieros, así como actividades educacionales y culturales del más alto nivel; aspectos que ubican a este tipo de ciudades globales como las más importantes en la jerarquía urbana a nivel mundial.

Sin dejar de reconocer que Nueva York, Londres y Tokio se proponen como las ciudades insignia de este proceso, no hay que dejar de lado la existencia de otras metrópolis de menor importancia, y por tanto de menor jerarquía, que también se reconocen como parte de esta red global de ciudades. En ese sentido Marcuse y Van Kempen (2000) proponen que independientemente de su nivel de desarrollo diversas ciudades del planeta comenzaron a desempeñar funciones globales y con ello una transformación hacia ciudades en vías de globalización sin ser estrictamente, en el sentido en que lo propone Sassen, ciudades globales; para el caso de Latinoamérica se reconocen ciudades como

(3) El autor destaca que el cambio es muy posible que se derive más de factores propios del proceso de metropolización, en ese sentido endógeno, que del poder transformador del proceso de globalización; a lo anterior se le suma la consideración de que una gran parte de los cambios experimentados por las metrópolis aparecieron durante el periodo fordista y en ese sentido la inercia y temporalidad de los efectos ponen en duda que se deriven del proceso de globalización (DE MATTOS, 2002). 
Río de Janeiro a Brasil, Buenos Aires, Argentina, Santiago, Chile, Ciudad de México, entre otras. Estos autores al analizar el tema de los impactos urbanos de la globalización cuestionan el establecimiento de una relación entre esta última y la posición jerárquica de las ciudades, en el sentido de que solamente las ciudades de más alto orden se vean impactadas mientras que las ciudades de menor orden no; Marcuse y Van Kempen consideran que prácticamente todas las ciudades son tocadas por el proceso de globalización y que más que deberse a la posición jerárquica que ocupan las ciudades es más bien una consecuencia de la naturaleza y alcance de la influencia del proceso de globalización.

\section{Zonas metropolitanas del occidente de México}

Sin lugar a dudas los retos que enfrenta el desarrollo urbano de México en el siglo XXI están estrechamente relacionados con los cambios que a escala planetaria dan forma y contenido al proceso de globalización. En el caso de nuestro país el proceso de reestructuración económica, derivado de la apertura comercial, está provocando una distribución más diversificada de las actividades productivas en el territorio nacional y, con ello, observamos tanto la emergencia de nuevas tendencias como la consolidación de tendencias previas en la distribución poblacional y en el crecimiento e interacción de las ciudades.

En el marco de estas tendencias emergentes encontramos que hoy en día un número importante de ciudades en México rebasan los límites del municipio que originalmente las contuvo ampliándose sobre territorios de municipios vecinos creando conurbaciones, otro tipo de tendencia emergente es la creciente interrelación socioeconómica entre ciudades de municipios contiguos sin existir una continuidad física. En las anteriores situaciones existe un elemento en común que se define como un conglomerado urbano, más específicamente una Zona Metropolitana, cuya estructura involucra a dos o más circunscripciones político-administrativas de nivel municipal, existiendo casos en que los municipios integrantes pertenecen a dos estados, incluso países, diferentes y se define como una ciudad "grande" cuyos límites rebasan los de la unidad político administrativa que originalmente la contenía, para el caso de México la unidad es el municipio (NEGRETE; SALAZAR, 1986).

En ese sentido las zonas metropolitanas de México se caracterizan por configurar una extensión territorial que incluye la unidad político-administrativa de origen y las unidades contiguas, presentan procesos de suburbanización y se manifiesta en ellas una interrelación socioeconómica a través de centros de trabajo y de concentración de servicios; esta configuración "muestra más precisamente la dimensión real de la de la ciudad a que se refiere” (NEGRETE; SALAZAR, 1986, p. 99), y hoy sus límites se definen a partir de los propios límites de las delegaciones o municipios que la integran y por tanto difiere del concepto de área metropolitana o área urbana (GRAIZBORD; SALAZAR, 1986, p. 121).

Para el caso de México las zonas metropolitanas son los elementos de mayor jerarquía del sistema urbano nacional, y se les considera como un fenómeno de urbanización 
relativamente reciente que en los últimos años ha manifestado un acelerado crecimiento, de hecho para el 2000 existían en el país 39 zonas metropolitanas mayores de 100,000 habitantes en las que residía el 46\% de la población nacional (SEDESOL, 2001).

Con base en información del año 2000, proveniente del XII Censo General de Población y Vivienda del Inegi, se reconoce en el 2004 la existencia de 55 zonas metropolitanas que contribuyen con el $71 \%$ del PIB del país, y se les reconoce el potencial de incidir en el desarrollo económico y social de sus respectivas áreas de influencia; el conjunto de 55 zonas metropolitanas registro una población de 51.5 millones de habitantes en el año 2000, que representan cerca del 53\% de la población total del país (SEDESOL; CONAPO; INEGI, 2004).

En resumen, en los últimos 20 años el fenómeno metropolitano se ha intensificado y diversificado a tal grado que las 55 zonas metropolitanas las podemos clasificar por el tamaño de su población en aquellas con más de un millón de habitantes (9), las que tienen entre 500 mil y un millón de habitantes (14) y por último las que se ubican en el rango de 100 mil a 500 mil habitantes (32). Cabe resaltar que 33 (60 por ciento) de las zonas metropolitanas del país se localizan en la zona centro del país ${ }^{(4)}$, y de este número el 51.5 por ciento se ubican en la Región Centro Occidente (RCO) del país (ver Cuadro 1).

\section{Cuadro 1 - Población total y tasa de crecimiento por rango de población de las zonas metropolitanas, 1990-2000}

\begin{tabular}{|c|c|c|c|c|c|c|c|}
\hline \multirow[b]{2}{*}{ Rango (habitantes) } & Zonas & \multicolumn{3}{|c|}{ Población } & \multicolumn{3}{|c|}{ Tasa de crecimiento medio anual (\%) } \\
\hline & Metropolitanas & 1990 & 1995 & 2000 & 1990-1995 & $1995-2000$ & $1990-2000$ \\
\hline Total nacional & & $81,249,645$ & $91,158,290 !$ & $97,483,412$ & 2.3 & 1.4 & 1.8 \\
\hline Total nacional zonas metropolitanas & 55 & $4 \cdot 1,168,7134$ & $47,503,876$ & $51,502,972$ & 2.9 & 1.6 & 2.3 \\
\hline 1000000 o más & 9 & $27,092,926$ & $30,958,271$ & $33,501,764$ & 2.7 & 1.6 & 2.1 \\
\hline 500000 a 999999 & 14 & $7,529,899$ & $8,867,110$ & $9,697,976$ & 3.3 & 1.8 & 2.6 \\
\hline 100000 a 499999 & 32 & $6,545,888$ & $7,678,495$ & $8,303,232$ & 3.2 & 1.6 & 2.4 \\
\hline \multicolumn{8}{|l|}{ Zonas Metropolitanas Centro del } \\
\hline País & 33 & $29,738,183$ & $33,902,134$ & $36,438,667$ & 2.7 & 1.5 & 2.1 \\
\hline 1000000 o más & 5 & $22,055,530$ & $24,911,218$ & $26,702,114$ & 2.5 & 1.4 & 1.9 \\
\hline 500000 a 999999 & 7 & $3,745,131$ & $4,462,729$ & $4,897,041$ & 3.6 & 1.9 & 2.7 \\
\hline 100000 a 499999 & 21 & $3,937,522$ & $4,528,187$ & $4,839,512$ & 2.8 & 1.3 & 2.1 \\
\hline \multicolumn{8}{|l|}{ Zonas Metropolitanas Región } \\
\hline Centro Occidente & 17 & $7,987,387$ & $9,342,064$ & $10,077,372$ & 3.2 & 1.5 & 2.4 \\
\hline 1000000 o más & 2 & $3,986,918$ & $4,656,076$ & $4,968,315$ & 3.2 & 1.3 & 2.2 \\
\hline 500000 a 999999 & 4 & $2,288,341$ & $2,713,722$ & $3,005,625$ & 3.5 & 2.1 & 2.8 \\
\hline 100000 a 499999 & 11 & $1,712,128$ & $1,972,266$ & $2,103,432$ & 2.9 & 1.3 & 2.1 \\
\hline
\end{tabular}

Fuente: Elaboración propia con base a información proveniente de SEDESOL; CONAPO; INEGI (2004) “Delimitación de las zonas metropolitanas de México".

(4) En torno al eje neovolcánico que es una franja volcánica del territorio nacional que cruza el centro del país desde las costas del Pacífico hasta las costas del Golfo de México. 


\section{Mapa 1 - Ubicación de la Región Occidente de México}

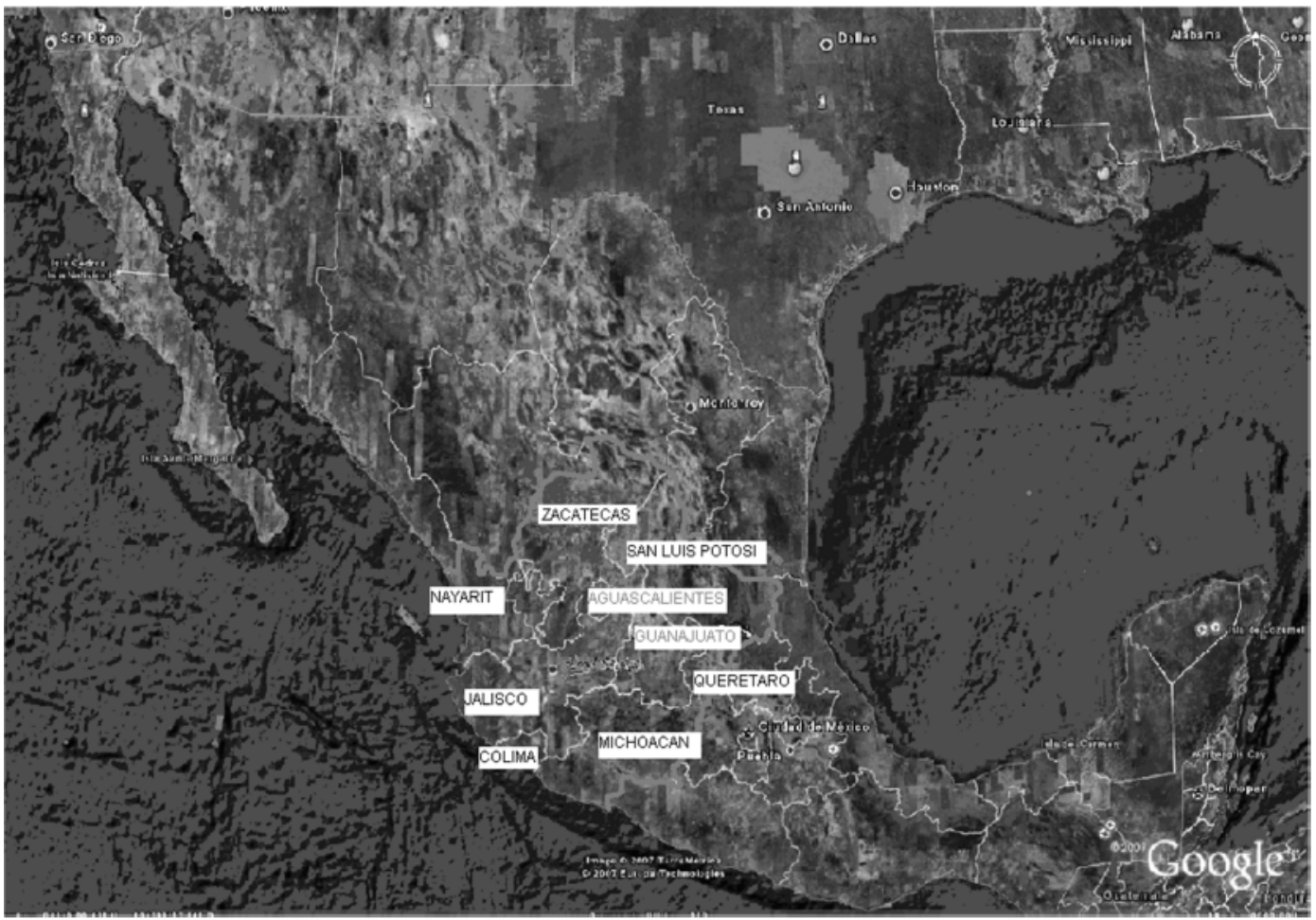

En el cuadro 1 se observa que en su conjunto las zonas metropolitanas del país contribuyen con parte del crecimiento poblacional nacional al registrar un crecimiento medio anual de 2,3 por ciento durante el periodo de 1990 al 2000. Parte de ese dinamismo se debe a la participación de las metrópolis de la RCO que registran un crecimiento medio anual de 2,4 por ciento, cifra por encima del experimentado por el país (1,8 por ciento) y por el conjunto de zonas metropolitanas del centro del país (2,1 por ciento).

Para el año 2000 de las 17 zonas metropolitanas presentes en la RCO, dos Guadalajara (ZMG) y León (ZML), contienen una población mayor a un millón de habitantes; por su parte 4, Aguascalientes (ZMA), Morelia (ZMM), Querétaro (ZMQ) y San Luis Potosí-Soledad de G. S. (ZMSLP), son ciudades con una población entre quinientos mil y un millón de habitantes; por último las zonas metropolitanas con una población entre cien mil y quinientos mil habitantes son 11, Colima-Villa de Alvarez (ZMCVA), Tecomán (ZMT), Moroleón-Uriangato (ZMMU), San Francisco del Rincón (ZMSFR), Ocotlán (ZMO), Puerto Vallarta (ZMPV), Zamora-Jacona (ZMZJ), La Piedad (ZMLP), Tepic (ZMT), Río Verde-Ciudad Fernández (ZMRVCF) y Zacatecas (ZMZ) (ver Cuadro 2). 


\section{Cuadro 2 - Zonas Metropolitanas del Occidente de México}

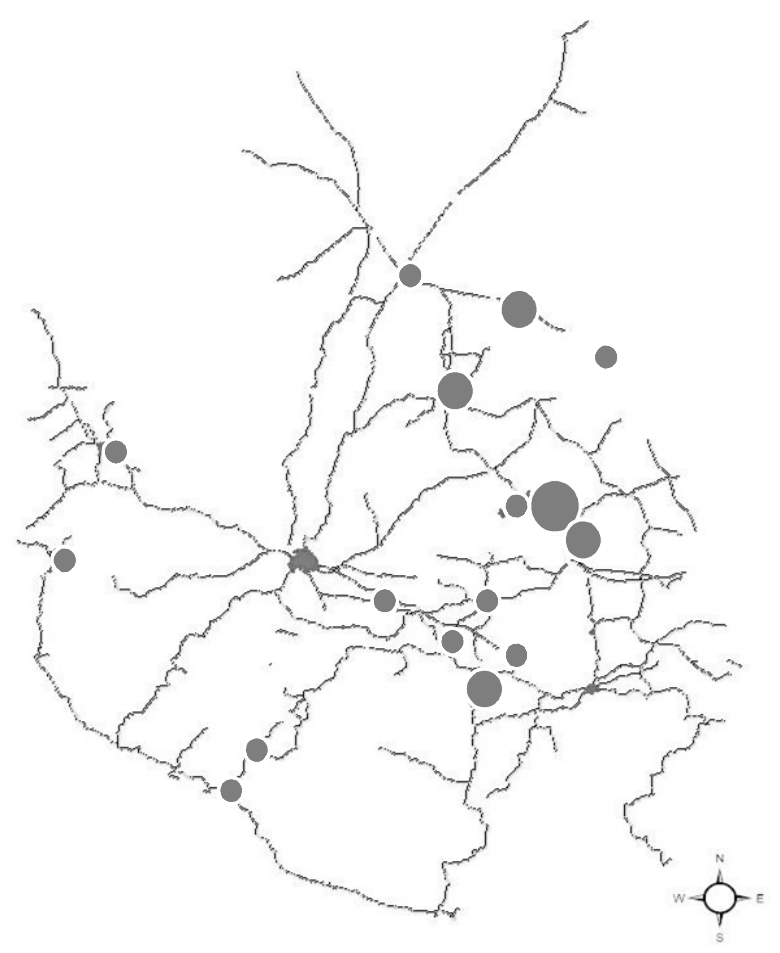

ZONAS METROPOLITANAS DEL OCCIDENTE DE MÉXICO

1 AGUASCALIENTES

2 COLIMA - VILLA DE ALVAREZ

3 GUADALAJARA

4 LEON

5 MORELIA

6 MOROLEÓN

7 OCOTLAN

8 LA PIEDAD

9 PUERTO VALLARTA

10 QUERETARO

11 RÍO VERDE-CIUDAD FERNANDEZ

12 SAN FRANCISCO DEL RINCÓN

13 SAN LUIS POTOSÍ-SOLEDAD DE G. S.

14 TECOMÁN

15 TEPIC

16 ZACATECAS-GUADALUPE

17 ZAMORA-JACONA

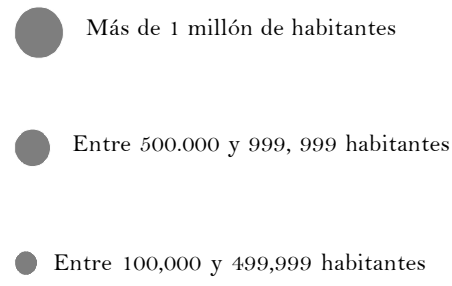

Fuente: Elaboración propia.

En el Cuadro 3 podemos observar las 17 zonas metropolitanas de la RCO en cuanto su población total, sus tasas de crecimiento y la superficie en kilómetros cuadrados. 


\section{Cuadro 3 - Población total, tasa de crecimiento y superficie por zona metropolitana de la Región Centro-Occidente de México, 1990-2000}

\begin{tabular}{|c|c|c|c|c|c|c|c|c|}
\hline \multirow{2}{*}{$\begin{array}{c}\text { Zonas Metropolitanas } \\
\text { Región Centro-Occidente }\end{array}$} & \multirow[t]{2}{*}{$\begin{array}{l}\text { Entidades } \\
\text { federativas }\end{array}$} & \multicolumn{3}{|c|}{ Población } & \multicolumn{3}{|c|}{ Tasa de crecimiento medio anual (\%) } & \multirow{2}{*}{$\begin{array}{c}\text { Superficie } \\
\mathrm{Km}^{2}\end{array}$} \\
\hline & & 1990 & 1995 & 2000 & $1990-1995$ & $1995-2000$ & $1990-2000$ & \\
\hline $\begin{array}{l}\text { ZM de Aguascalientes } \\
\text { ZM de Colima-Villa de }\end{array}$ & Aguascalientes & 547,366 & 637,303 & 707,516 & 3.1 & 2.1 & 2.6 & 1,685 \\
\hline Alvarez & Colima & 154,347 & 187,081 & 210,766 & 3.9 & 2.4 & 3.2 & 1,033 \\
\hline $\begin{array}{l}\text { ZM de Tecomán } \\
\text { ZM de Moroleón- }\end{array}$ & Colima & 110,481 & 119,051 & 127,863 & 1.5 & 1.4 & 1.5 & 1,201 \\
\hline Uriangato & Guanajuato & 94,901 & 96,545 & 100,063 & 0.3 & 0.7 & 0.5 & 273 \\
\hline $\begin{array}{l}\text { ZM de León } \\
\text { ZM de San Francisco del }\end{array}$ & Guanajuato & 983,0501 & $, 173,659$ & $1,269,179$ & 3.6 & 1.6 & 2.6 & 1,767 \\
\hline Rincón & Guanajuato & 114,034 & 132,048 & 145,017 & 3.0 & 1.9 & 2.4 & 710 \\
\hline ZM de Guadalajara & Jalisco & $3,003,8683$ & 482,417 & $3,699,136$ & 3.0 & 1.2 & 2.1 & 2,734 \\
\hline ZM de Ocotlán & Jalisco & 101,905 & 115,021 & 125,027 & 2.5 & 1.7 & 2.1 & 1,089 \\
\hline ZM de Puerto Vallarta & Jalisco-Nayarit & 151,288 & 196,953 & 244,536 & 5.4 & 4.4 & 4.9 & 1,472 \\
\hline $\begin{array}{l}\text { ZM de Zamora-Jacona } \\
\text { ZM de La Piedad }\end{array}$ & $\begin{array}{l}\text { Michoacán } \\
\text { Michoacán- }\end{array}$ & 185,445 & 214,938 & 216,048 & 3.0 & 0.1 & 1.5 & 460 \\
\hline & Guanajuato & 219,004 & 229,716 & 229,372 & 1.0 & 0.0 & 0.5 & 1,846 \\
\hline ZM de Morelia & Michoacán & 526,772 & 614,698 & 659,940 & 3.1 & 1.4 & 2.3 & 1,456 \\
\hline ZM de Tepic & Nayarit & 268,185 & 327,375 & 342,840 & 4.1 & 0.9 & 2.5 & 2,160 \\
\hline $\begin{array}{l}\text { ZM de Querétaro } \\
\text { ZM de Río Verde-Ciudad }\end{array}$ & Querétaro & 555,491 & 679,757 & 787,341 & 4.1 & 3.0 & 3.5 & 1,650 \\
\hline $\begin{array}{l}\text { Fernández } \\
\text { ZM de San Luis Potosí- }\end{array}$ & San Luis Potosí & 121,212 & 127,273 & 128,935 & 1.0 & 0.3 & 0.6 & 3,596 \\
\hline -Soledad de G. S. & San Luis Potosí & 658,712 & 781,964 & 850,828 & 3.5 & 1.7 & 2.6 & 1,742 \\
\hline $\begin{array}{l}\text { ZM de Zacatecas } \\
\text { Total zonas }\end{array}$ & Zacatecas & 191,326 & 226,265 & 232,965 & 3.4 & 0.6 & 2.0 & 1,263 \\
\hline metropolitanas & & $7,987,3879$ & $, 342,0641$ & $0,077,372$ & 3.2 & 1.5 & 2.4 & 26,137 \\
\hline
\end{tabular}

Fuente: Elaboración propia con base a información proveniente de SEDESOL, CONAPO, INEGI (2004) "Delimitación de las zonas metropolitanas de México”.

\section{Mapa 2 - Zonas Metropolitanas en la Región Occidente de México}

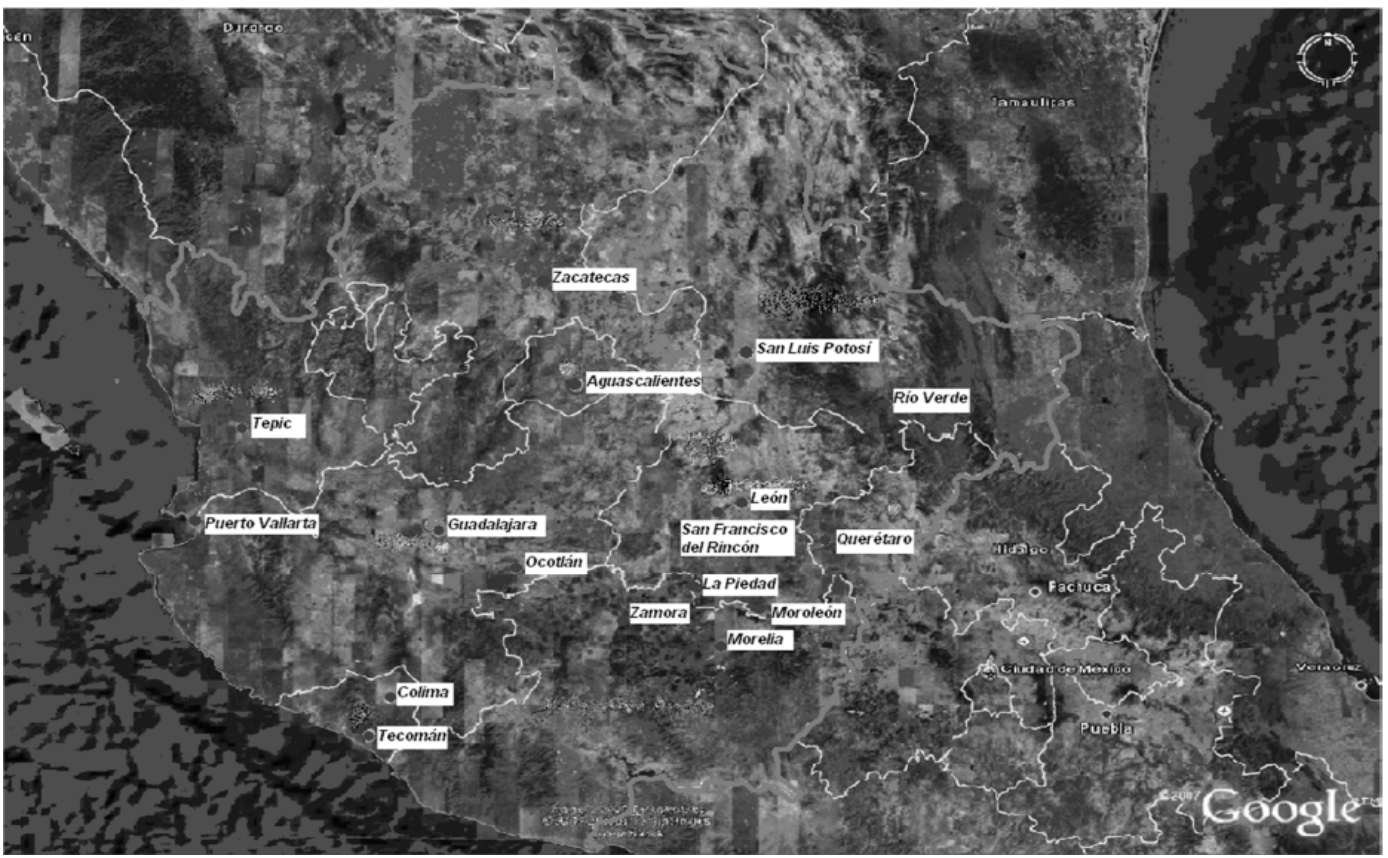




\section{A MANERA DE CONCLUSIÓN}

Las zonas metropolitanas del Occidente de México, como otras urbes del país, han venido enfrentando en los últimos 30 años una serie de cambios relacionados en gran medida con el acelerado proceso de integración al mercado mundial, en esta situación las ciudades compiten entre ellas en diferentes escenarios como el local, regional, nacional e internacional; intensificándose y otorgándole, en muchos de los casos a este último escenario una preponderancia que hace pensar que si no es una ciudad inserta en el proceso de globalización simple y llanamente se esta fuera de toda posibilidad de crecimiento e incluso de permanencia como tal.

Este tipo de apuesta permea la mayoría de nuestras ciudades y ello se refleja en las acciones más "significativas" que las administraciones de éstas realizan y en las que se suele considerar como más innovadoras a las estrategias que estén relacionadas con el marketing territorial, con acciones de impulso a la competitividad, como quiera que esta se entienda, y con lo que se ha denominado como gestión estratégica de las ciudades.

Sin embargo existe evidencia de que no solamente las ciudades que realizan acciones relacionadas con la globalización y la competitividad cuentan con posibilidades de ser ciudades con expectativas favorables no solo en lo económico, sino también en lo social, político y ambiental, esto es de ser y permanecer como ciudad en el transcurso del tiempo a pesar de las modas por ciertos tipos de estrategias de planeación urbana.

En ese sentido y atendiendo nuestro objetivo principal de ofrecer una agenda de investigación para las zonas metropolitanas del occidente del país presentamos a continuación algunas ideas en las cuales podríamos basar nuestros futuros estudios.

\section{Metropolización Y GLOBALIZACión}

Considerando la importancia que la reestructuración económica esta teniendo en nuestras ciudades, es necesario analizar las tendencias y pautas emergentes en el proceso de urbanización-metropolización que se desarrollan en el marco de los cambios en la economía internacional. En ese sentido es importante considerar que la reestructuración económica (productiva y del consumo) a través de la apertura comercial sin lugar a dudas presenta manifestaciones territoriales como la redistribución espacial de la industria y de la población, además de propiciar cambios en la articulación de las relaciones económicas en y entre las regiones, así como la reestructuración de los sistemas de las ciudades y con ello de la jerarquía urbana. Lo anterior no es una tarea sencilla sobre todo si reconocemos la necesidad de distinguir entre los fenómenos resultantes de factores exógenos ligados al proceso de reestructuración económica y los que son parte de tendencias endógenas, ya sean de carácter nacional, regional o local.

\section{AnÁlisis de la baSe ECONómica de laS CIUDADES}

Otro elemento de singular interés lo constituye la necesidad de abundar en el papel económico que tienen las ciudades, esto es, tomando en cuenta la aparición de nuevas 
perspectivas de desarrollo, a través de la apertura comercial, se hace necesario analizar como funcionan y crecen las ciudades. En ese sentido y atendiendo los cambios experimentados por las ciudades en su organización económica, entonces debemos abordar aspectos como las principales modificaciones que están experimentando las actividades productivas eminentemente urbanas como son la industria, el comercio y los servicios.

\section{Gestión METROPOLITANA}

Los municipios que integran las zonas metropolitanas en nuestro país, presentan generalmente diferencias en cuanto a su oferta de bienes y servicios, así como en sus características ambientales y en sus costos de producción. Esta diferenciación se le puede utilizar como una ventana de oportunidad en la atracción de empresas, residentes y visitantes, que contribuyan al incremento en los ingresos, tanto en los municipios de manera individual como en el conjunto de ellos que constituyan las zonas metropolitanas; en ese sentido la competencia entre municipios puede significar el avance de unos y el deterioro de otros, lo anterior nos permite señalar la necesidad de promover estudios que propicien la búsqueda de acuerdos cuyo objetivo principal sería el potenciar la competitividad de los municipios de manera conjunta, mediante estrategias de cooperación. Sin lugar a dudas lo anterior requiere identificar estrategias y tipos de arreglos institucionales, esto es, la creación de gobiernos metropolitanos.

Por último queremos aclarar que la lista de temas de interés en el análisis de las zonas metropolitanas sin lugar a dudas debería ser mucho más amplia de lo que aquí hemos señalado y precisamente ello se constituye en parte del trabajo pendiente ya que la presente no es más que una propuesta de agenda de investigación para las zonas metropolitanas del Occidente de México.

\section{BibliogRAfía}

DE MATTOS, Carlos A. Metropolización y suburbanización. EURE (Santiago). (online) mayo 2001, v. 27 , n. 80, 2001. p.5-8. Disponible en: <http://www: <http://www.scielo.cl/scielo.php? script=sci_arttext\&pid=So250-71612001008000001\&lng= es\&nrm=iso $>$ Acesso em: 18.8.2007.

. Transformación de las ciudades latinoamericanas: ¿Impactos de la globalización? EURE (Santiago). (online). v. 28 , n.85 p.5-10, dic. 2002. Disponible en: <http://www.scielo.cl/ scielo.php? script $=$ sci_arttext\&pid=S0250-7161200200850000 $1 \& \operatorname{lng}=$ es\&nrm=iso $>$ Acesso em: 18.8.2007.

DÍAZ, Verónica L. Competencia-colaboración en el mapa mundial de ciudades. En: GÓNZALEZ, Daniel; ORENDAIN, Tomas; GARCÍA, Maria Luisa (edits.) Ciudad y urbanismo: ideas y procesos. México: Universidad de Guadalajara, 2002.

GRAIZBORD, Boris; HÉCTOR, Salazar. Expansión física de la ciudad de México. En: GARZA, Gustavo (coord.). Atlas de la ciudad de México. México: Departamento del Distrito Federal/El Colegio de México, 1986. 
MARCUSE, Peter; KEMPEN, Ronald Van. Globalizing cities. A new spatial order? Cambridge: Blackwell, 2000.

MARKUSEN, Ann; SCHROCK, Greg. The distinctive city: divergent patterns in growth, hierarchy. Urban Studies, v. 43, n. 8, julio 2006.

MIRET, Naik. Las aportaciones de la inmigración al proceso de metropolización: el caso de Barcelona. Scripta Nova (Barcelona). (online), v. 72, n. 94, p. 1-12, Ago. 2001. Disponible en: < http://www.scielo. $\mathrm{cl} /$ scielo.php?script $=$ sci_arttext\&pid=So250-71612003008800001\&lng $=$ es\&nrm $=$ iso $>$ Acesso em: 18.8.2007.

NEGRETE, María. Eugenia; SALAZAR, Héctor. Zonas metropolitanas en México, 1980. Estudios Demográficos y Urbanos, v. 1, n. 1, 1986.

OLIVARES, Adriana; GÓNZALEZ, Daniel. Nuevos territorios urbanos: Consideraciones de la espacialidad contemporánea. Urbano, Universidad de BíoBío, v. 7, n. 9, mayo 2004.

RODRÍGUEZ, Juan J. La nueva economía y sus efectos en la ciudad de Guadalajara: la última década del siglo XX. México: Universidad de Guadalajara, 2006.

SASSEN, Saskia. The global city. 2. ed. New York, London, Tokio. New York: Princeton, 2001.

SEDESOL. Intervención de la Secretaria de Desarrollo Social en "25 Período Extraordinario de Sesiones de la Asamblea General de las Naciones Unidas para realizar un Examen y una Evaluación Generales de la Aplicación de los Resultados de la Conferencia de las Naciones Unidas sobre los Asentamientos Humanos (HABITAT II), celebrado en Nueva York el 6 de junio del 2001. Disponible en: <http://www.un.org/ga/habitat/ statements/docs/mexicoS.html>.

UNIKEL, Luis; CRESCENCIO, Ruiz; GARZA, Gustavo. El desarrollo urbano en México. Diagnóstico e implicaciones futuras. México: Colegio de México, 1978. 